Patrick D. O.Connor, Lyle N. Long, and James B. Anderson, "The Direct Simulation of Detonations," Invited Paper, AIAA Paper No. 2006-4411, AIAA/ASME/SAE/ASEE Joint Propulsion Conference, Sacramento, CA, July, 2006.

\title{
The Direct Simulation of Detonations
}

\author{
Patrick D. O’Connor ${ }^{*}$, Lyle N. Long ${ }^{\dagger}$, and James B. Anderson ${ }^{\ddagger}$ \\ Pennsylvania State University, University Park, PA, 16802
}

\begin{abstract}
In this paper we review the status of the simulation of detonations using the direct simulation Monte Carlo method and report on progress in applications to the hydrogenchlorine system. Recent work with this stochastic approach has demonstrated successes in treating simple model systems, confirmed many of the details found in earlier analyses, and revealed new phenomena such as ultrafast detonations. Current efforts are directed toward simulations for fully realistic kinetic systems. These require a more elaborate and detailed treatment of dissociation and recombination reactions as well as energy exchanges involving many degrees of freedom and discrete vibrational levels. Initial results are encouraging and indicate such calculations are feasible for complex reaction systems.
\end{abstract}

\section{Introduction}

$\mathrm{T}$ HE modern necessity for accurate computational methods for gas phase combustion modeling has made the treatment of kinetic systems an important challenge. Numerical methods spanning both continuum and particle based regimes have been used to model systems containing detonations. A detonation is a wave traveling at supersonic velocity in a gaseous medium, and it is driven by the energy released from the exothermic reactions within the wave. The continuum Navier-Stokes (NS) and Euler equations approximate the detonation front as a discontinuity of zero thickness, which may be acceptable if one is only concerned with the upstream or downstream conditions. The direct simulation Monte Carlo (DSMC) method is a stochastic method that can accurately calculate the upstream and downstream conditions of the system, as well as correctly simulate the details of the detonation wave structure given the appropriate level of grid resolution. The DSMC method has been used extensively to study the characteristics of one-dimensional detonations. ${ }^{1-4}$ The continued development of our transient, multidimensional $\mathrm{C}++$ program has led to the current investigation into complex explosive mechanisms.

\section{A. Brief History}

The history of detonation includes a small number of important scientific "firsts" in studies of the fundamental nature of the uniform one-dimensional detonation. In 1881 the first experimental work on supersonic flame propagation involving the recognition of detonation was completed independently by two pairs of French chemists, Mallard and Le Chatelier ${ }^{5}$ and Berthelot and Vieille. ${ }^{6}$ They discovered that specific mixtures of gases consistently produced distinct detonation velocities. Their work predates the first widely accepted theoretical treatment governing detonation phenomena by nearly two decades. Prior to their discoveries, scientists had been unable to explain the observed uniform supersonic propagation by means of diffusion and thermal conductivity. The discovery of detonation is commonly credited to Mallard and Le Chatelier as they were the first to explain that the supersonic velocities observed in detonations were achieved primarily through gas compression.

In 1899 D. L. Chapman ${ }^{7}$ established the theory of detonation based on the conservation of mass, momentum and energy across a detonation front approximated as a planar discontinuity. Appreciable contributions were made in published works in 1905 and 1906 by E. Jouguet ${ }^{8}$ who developed the simple theoretical treatment of onedimensional detonations as it is known today. The Chapman-Jouguet (CJ) theory allows for the determination of the velocity of a one dimensional steady state detonation by applying the conservation equations across the discontinuity and assuming the velocity of the burned gas is sonic (equal to the velocity of sound in the gas).

The CJ theory was the primary theoretical model used to study detonation until the 1940 s when Zel'dovich, ${ }^{9}$ von Neumann, ${ }^{10}$ and Döring ${ }^{11}$ independently proposed several modifications to alleviate deficiencies in the CJ theory.

\footnotetext{
* NSF Graduate Fellow, Department of Chemistry, 104 Chemistry Building, Member AIAA.

${ }^{\dagger}$ Professor, Aerospace Engineering, 229 Hammond Building, Fellow AIAA.

${ }^{*}$ Evan Pugh Professor, Department of Chemistry, 104 Chemistry Building.

Copyright $\mathbb{C}$ by L. N. Long. Published by the American Institute of Aeronautics and Astronautics with permission.
} 
One of the most significant developments of the Zel'dovich-von Neumann-Döring (ZND) theories was the introduction of thickness to the detonation front by means of a series of Hugoniot curves representing "successive fractions" of a reaction in a detonation. This modification replaced the single Hugoniot curve approach for describing pressure and density changes in the $\mathrm{CJ}$ theory and the underlying assumption of an infinitely fast reaction. Another important contribution was the validation of the CJ condition (sonic burned gases) based on stability arguments assuming one-dimensional behavior. It was determined that when the shock region preceded the reaction region, the detonation velocity was equal to the sum of the flow velocity and the speed of sound in the burned gas. The contributions by Zel'dovich, von Neumann, and Döring opened detonation theory to others to investigate the complex reaction-shock dynamic.

In 1958 Hirschfelder and Curtiss ${ }^{12}$ published a detailed study of temperature, pressure and composition as a function of distance in a steady state, gas phase detonation wave. They were the first to use the complete set of hydrodynamic relations, including coefficient of diffusion, viscosity and realistic relations for chemical kinetics. Hirschfelder and Curtiss were also very interested in the strong coupling between the shock and reaction regions. They considered examples of strong shock and reaction region coalescence and discovered that the waves lacked the characteristic von Neumann spike. Hirschfelder and Curtiss concluded that ..."if the Mach number is greater than unity, the solutions have an entirely different nature and exist only for a single ambient pressure rather than for a range of pressures."

These noted contributions have allowed us a standardized approach to the study of the formation and propagation of a one-dimensional detonation. It will be shown that much of our work using the direct simulation Monte Carlo method has followed closely these scientific contributions and provided confirmation at the molecular level.

\section{B. Computational and Theoretical Approaches}

There is a wide range of computational and theoretical approaches available to study fluid dynamic processes such as detonation. Figure 1 illustrates how fluid dynamic processes can be viewed as points in a 3-D space based upon the Knudsen number, the Mach number, and the Strouhal number. The Knudsen number $(\mathrm{Kn})$ is defined as the ratio of the mean-free-path to characteristic length scale $(\lambda / L)$, and is a measure of the nonequilibrium or viscous effects. The Knudsen number can be shown to be roughly inversely proportional to the Reynolds number $(U L / v)$, where $U$ and $v$ are the macroscopic flow velocity and kinematic viscosity, respectively. The Mach number is the ratio of the flow velocity to the speed of sound $(U / a)$, and is a measure of compressibility, including the incompressible, subsonic, transonic, supersonic, and hypersonic regimes. The Strouhal number is a nondimensional frequency $(f L / U)$ where $f$ is the frequency, and is a measure of unsteadiness in the flow. The Strouhal number ranges from steady-state flow to highly unsteady flow such as that encountered in acoustics or turbulence. Special cases of fluid dynamic processes (e.g., steady state flow, incompressible flow, or inviscid flow) can be represented as two-dimensional regions in this space. A detonation represents one of the most complicated fluid flows possible, since it involves large Knudsen numbers, large Mach numbers, and (often) unsteady behavior. The wide range of physical scales and phenomena involved in fluid dynamics is one of the reasons why there are many different computational approaches used. No single method can efficiently and effectively capture all the different aspects of all fluid dynamic flows. This situation is even more complicated when chemical reactions are involved.
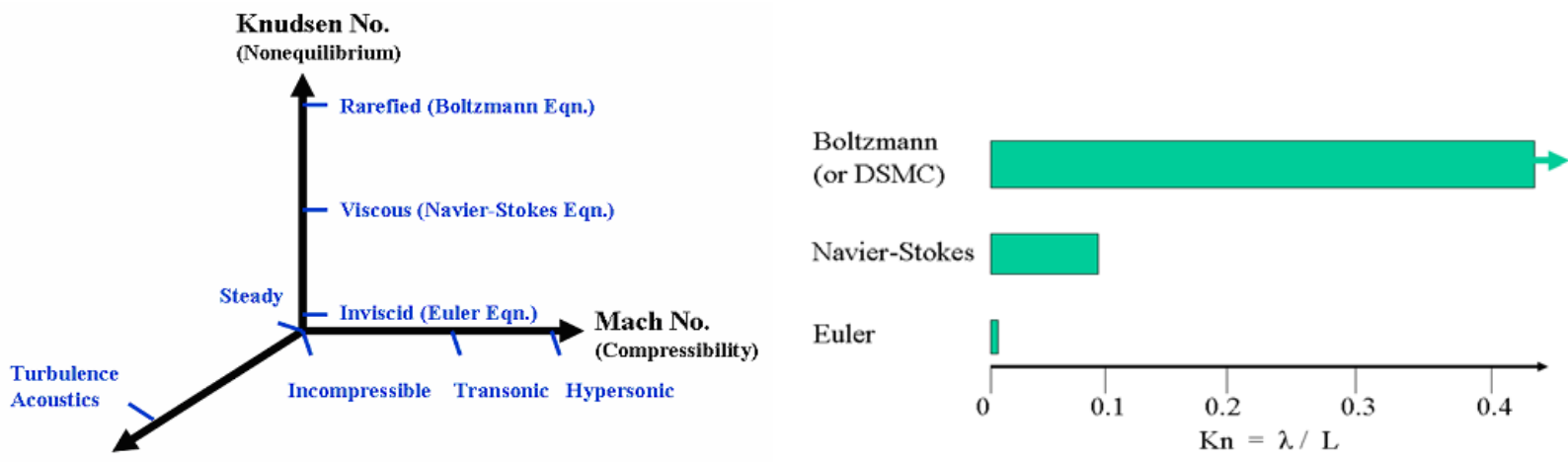

Strouhal No. (Unsteadiness)

Figure 1. 3-D schematic of fluid dynamic processes.

Figure 2. Validity of numerical methods based on a Knudsen number scale. 
The detonation process is highly nonlinear, so the simplest fluid dynamic model possible is one that uses the Euler equations. These continuum equations include the (inviscid) continuum mass, momentum, and energy equations which are four-dimensional (space and time) nonlinear partial differential equations based on the assumption that gas is locally in equilibrium (i.e., the particle velocity distribution is Maxwellian). Therefore, the Euler equations essentially specify a Knudsen number of zero, as shown in Fig. 2. The Navier-Stokes equations include more physics than the Euler equations, but are only capable of modeling flows with small Knudsen numbers $(\mathrm{Kn}<\sim 0.05)$. Chapman and Enskog ${ }^{13}$ showed how the Navier-Stokes equations could be derived from the Boltzmann equation if one assumes that $\mathrm{Kn}$ is very small. One must be careful not to use the Navier-Stokes equations in regimes where they are not applicable. ${ }^{14}$ The Boltzmann equation is valid for all Knudsen numbers, and the direct simulation Monte Carlo method effectively solves the Boltzmann equation. ${ }^{15,16}$ DSMC is much more effective than typical numerical methods for solving the Boltzmann equation due to the enormous number of degrees of freedom and the difficulty in including multiple species in the Boltzmann approach.

For detonations the characteristic length scale is the thickness of the detonation front (shock region) which typically varies between two and three mean-free-paths. This gives a Knudsen number range of approximately $0.3-$ 0.5 , far beyond that which the Euler and Navier-Stokes equations are capable of modeling. For Kn values larger than 0.1 the momentum and heat fluxes in the Navier-Stokes equations cannot be written in terms of macroscopic quantities and the equations are therefore incomplete, particularly when multiple species are involved. However, particle methods such as DSMC (or the Boltzmann equation) are valid for all Knudsen numbers (but are best suited for flows where $\mathrm{Kn}>0.05$ ). Therefore, applied to a detonation wave, the Navier-Stokes equations may be capable of computing the macroscopic flow properties of the unburned and burned gases (initial and final states) but would not be able to accurately model the steep temperature and density gradients found in the transition region of the detonation front.

The remainder of this paper will discuss the details of the DSMC approach and its application to kinetic systems. In Section II, the DSMC method will be introduced; discussing its range of applications, its usefulness to detonation simulations, and its advantages over continuum-based Euler and Navier-Stokes calculations. Section III will discuss modification and improvements to the fundamental DSMC program, including phenomenological energy exchange, classical and quantum rotational and vibrational modes, and the details of the kinetics used. Section IV will discuss an application of the DSMC method, the simulation of a hydrogen-chlorine explosion.

\section{Direct Simulation Monte Carlo}

The direct simulation Monte Carlo method is a stochastic approach used to model real gas flows on the molecular scale by simulating systems containing millions or billions of representative particles. DSMC provides the full details of the coupled gas dynamic and reaction effects and is capable of accurately representing the microscopic properties found in strong shocks or detonations. The DSMC method was introduced in 1963 by G. A. Bird ${ }^{17,18}$ and has since been applied extensively in the aerospace engineering field for rocket plume modeling, ${ }^{19}$ high altitude reentry problems, ${ }^{20}$ and shock/blast wave simulations. ${ }^{21}$ More recently, DSMC has been implemented in a variety of fields including astrophysics for studying planetary atmospheres, ${ }^{22}$ mechanical engineering for nanoscale flows, ${ }^{23,24}$ and in acoustics to study molecular absorption and sound wave propagation. ${ }^{25,26}$

A standard DSMC algorithm consists of five principal steps required for the simulation of chemically reactive gases, beginning with the initialization of the cells and particles at the start of each ensemble. A cell is a three dimensional computational domain in physical space that will initially contain some random fraction of the total number of particles specified for the simulation. Typically, each cell has dimensions smaller than the mean-free-path and a time-step size that is a small fraction of the mean collision time. After the initialization of the cells, molecules are randomly introduced into the domain and the following four uncoupled routines are repeated for every time step:

1) Move particles according to the time-step size $(d t)$ and their velocity, and apply boundary conditions.

2) Sort the particles into cells based on their positions relative to their local domain (cell).

3) Collide randomly selected particles and give them the opportunity for reaction and/or redistribution of energy.

4) Sample microscopic properties in each cell (after a specified number of time steps).

Macroscopic flow properties are calculated after a specified number of microscopic samples have been collected. Global properties such as temperature, velocity, pressure, and density can be calculated along with individual species properties such as average internal (rotation or vibration) energies, concentration profiles, etc.

An object-oriented programming approach was used in the development of this DSMC program. This approach allows the DSMC algorithm to be divided into physical objects that are individually maintained. Cell and Particle classes were created to govern fundamental components of the algorithm. The Cell class governs several methods 
(functions) that would occur inside a cell, such as collisions and the sampling of microscopic properties. Each 'cell' object created has its own dimensions and contains 'atom' objects. The Particle class creates 'atom' objects that are responsible for recording their positions and velocities. This data is private, and it can be sent to objects outside the Particle class, but cannot be altered. For example, for sorting purposes a cell can request an atom's position in space, but cannot modify its position because that is information held privately by the atom. Through inheritance, polymorphism and encapsulation it was possible to develop a $\mathrm{C}++$ program that is significantly easier to read, maintain, and modify than conventional C or FORTRAN procedural-based programs.

\section{A. Research Background}

Our work using the DSMC method to model detonations began with the simulation of steady one dimensional detonation waves having Chapman-Jouguet velocities. ${ }^{1}$ To clarify the results and avoid any extraneous errors, a simple bimolecular reaction of the type $\mathrm{A}+\mathrm{M} \rightarrow \mathrm{B}+\mathrm{M}$ was modeled, where $\mathrm{A}$ and $\mathrm{B}$ were identical species $(\mathrm{M}=$ A or B). The gases were specified as ideal and calorically perfect with constant heat capacities. The reaction crosssections calculated with a related steric factor and an energy dependence corresponding to the Arrhenius behavior of the reaction. DSMC calculations were carried out for a variety of conditions covering a wide range of reaction rate parameters and exothermicities.

Our early simulations of detonations provided complete details of the properties of the entire system as it varies across the detonation wave. Temperature, density, and reaction-rate peaks were separated and the dynamics of the wave front was analyzed. From these simulations, it was determined that temperature and density maxima depend strongly on reaction rate, and the thickness of the reaction zone is strongly coupled to the conditions of the system. The results served largely as a baseline test since one-dimensional detonations are well understood, and the inviscid 1-D conservation laws provide reasonable predictions of temperature jumps across the detonation and the detonation velocities. The temperature profile in Fig. 3 was calculated using the DSMC method and varies by more than 8,000 $\mathrm{K}$ over a distance of less than 2 micrometers. This temperature profile could not be modeled accurately using 1-D conservation laws. Figure 4 shows the excellent agreement between the DSMC results and CJ theory for detonation velocities as a function of exothermicity.

Extending this work, exothermic and endothermic reactions were also considered. ${ }^{2,3}$ These reaction systems yield pathological detonations that cannot be predicted using the CJ hypothesis. We found that the detonation velocity could be influenced significantly when endothermic and exothermic reactions were both present. By accurately studying the pathological detonation front, it was possible to show that DSMC calculations exhibited the behavior suggested by Kirkwood and $\mathrm{Wood}^{27}$ and by von Neumann ${ }^{10}$ for pathological detonations.

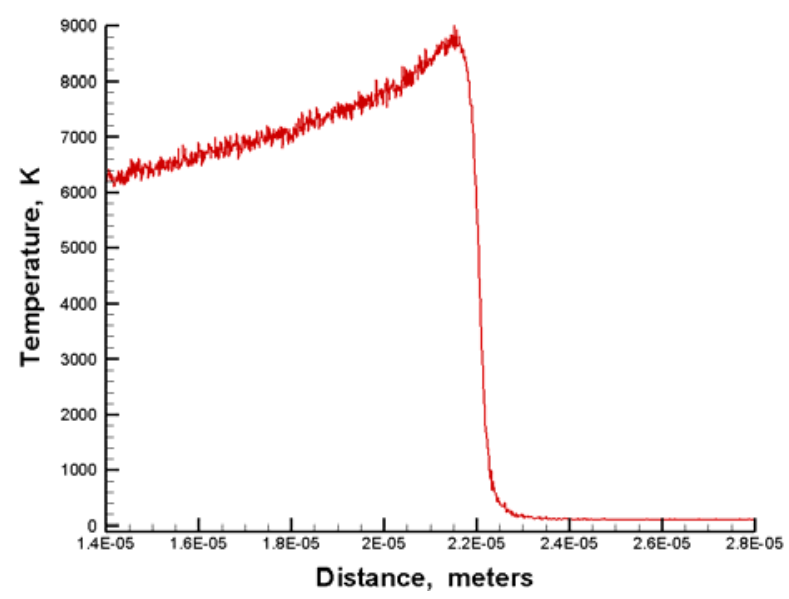

Figure 3. Detonation profile for an $\mathbf{A}+\mathbf{M} \rightarrow \mathbf{B}+\mathbf{M}$ type reaction using DSMC.

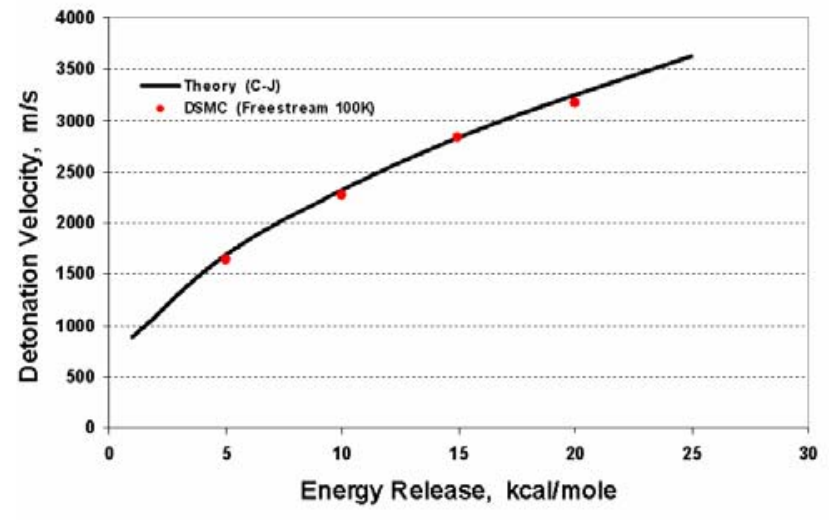

Figure 4. Detonation velocities as a function of energy release (exothermicity) for DSMC and CJ theory. 
Following the aforementioned research, a comprehensive investigation into the interaction between the shock and reaction regions was conducted. ${ }^{3}$ Adhering to the conditions of the ZND theory, simulations in which the shock preceded the reaction region yielded Chapman-Jouguet velocities as expected. However, for very fast reactions, the shock and reaction regions were found to overlap and the assumptions required for the CJ and ZND theories were no longer valid. The resulting detonation velocities were characterized as ultrafast, as they were found to exceed the steady-state velocities predicted by both CJ and ZND theories. Figure 5 illustrates how the ultrafast detonations exceeded the CJ velocities for very fast reactions (i.e., very low activation energies). The greatest velocity exceeded the CJ predicted velocity by roughly $6 \%$.

Continued investigation into the ultrafast regime involved the addition of a heavy non-reacting gas $\mathrm{C}$ into the previously described $\mathrm{A}+\mathrm{M} \rightarrow \mathrm{B}+\mathrm{M}$ detonating system. ${ }^{4}$ The addition of the non-reacting gas (with a molecular weight near that of Xenon) effectively lowers the speed of sound in the burned gas, and as a result the Chapman-Jouguet detonation velocity. Therefore, any ultrafast phenomena would have a much higher velocity relative to that of the

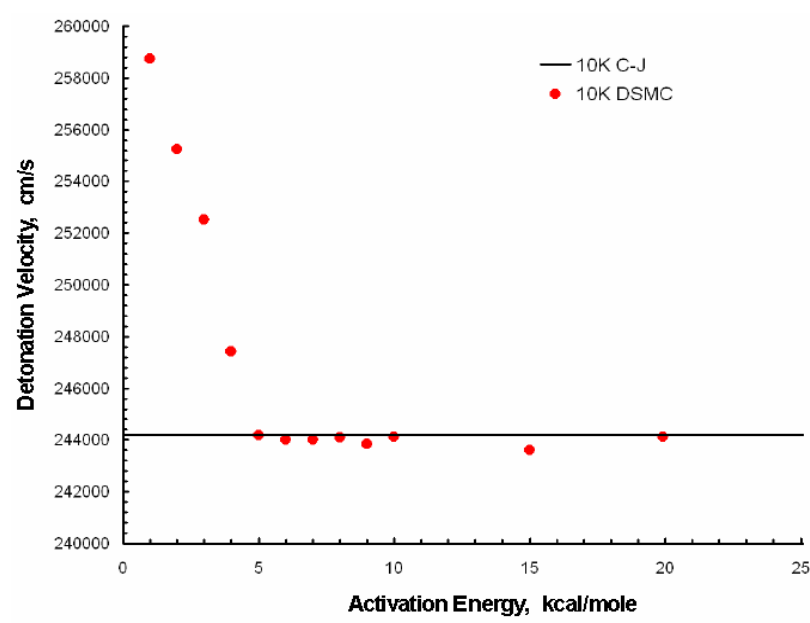

Figure 5. Variation in detonation velocity with activation energy. reduced CJ-predicted values. It was also determined that the addition of the nonreactive gas produced a stronger reaction-shock correlation, and resulted in the simulation of ultrafast detonations at much higher activation energies (slower reaction rates) than previously predicted. ${ }^{3}$ Figures 6 and 7 show temperature and species concentrations through the detonation waves for both slow and fast reactions with a $25 \%$ concentration of $\mathrm{C}$ particles. The activation energy $\left(\mathrm{E}_{\mathrm{a}}\right)$ in Fig. 6 is $10 \mathrm{kcal} / \mathrm{mole}$ and there is a distinct separation between the shock and reaction regions and it was again determined that these conditions yield CJ-predicted behavior. In Fig. 7, $\mathrm{E}_{\mathrm{a}}$ is $1 \mathrm{kcal} / \mathrm{mole}$ and a substantial overlap of shock and reaction regions produces detonation velocities exceeding CJ predictions by roughly $46 \%$. Under these conditions the largest deviation was calculated to be a $58 \%$ relative increase over CJ for an initial 50/50 mixture $\mathrm{A}$ and $\mathrm{C}$ particles.

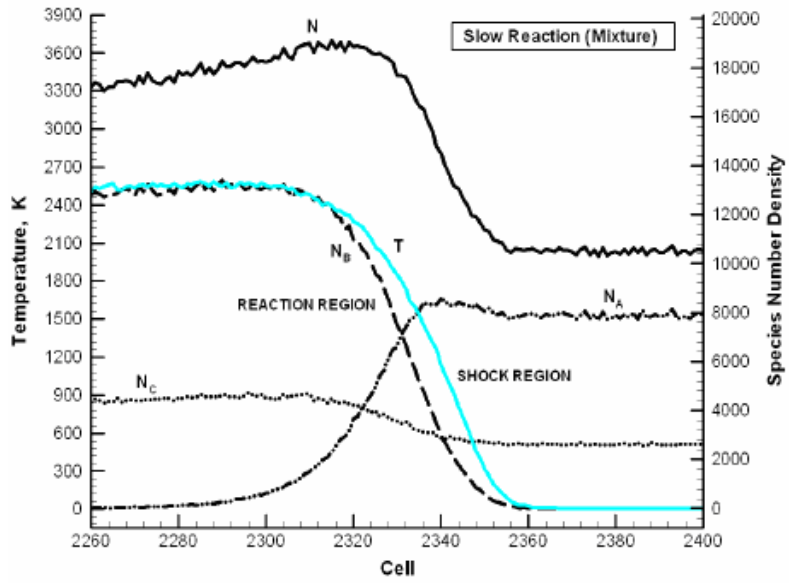

Figure 6. Separation of reaction and shock regions for normal detonation. ${ }^{4}$

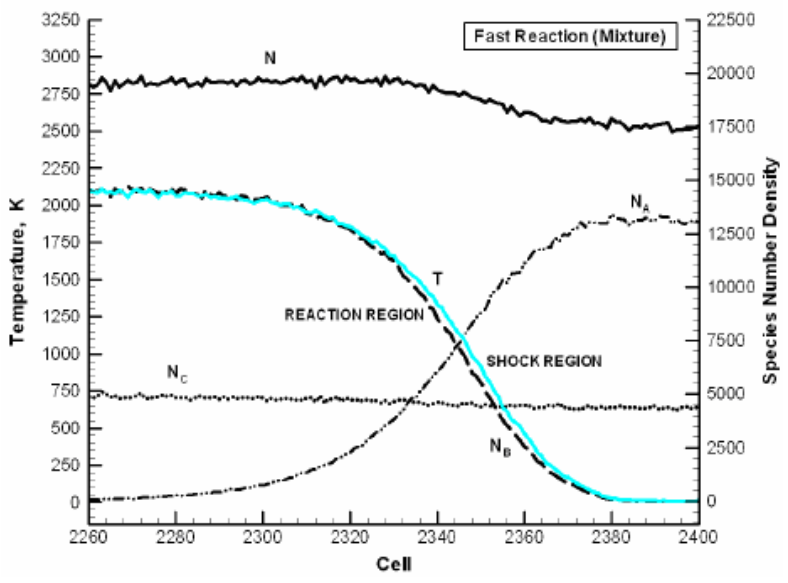

Figure 7. Coalescing of reaction and shock regions for ultrafast detonation. ${ }^{4}$

\section{B. DSMC Modifications}

Several modifications to our DSMC program have been introduced in order to improve the applicability of the simulations by making the molecules and their interactions more representative of those for typical detonation 
experiments. These modifications include a phenomenological energy distribution, discrete or continuous vibrational modes, and detailed reaction kinetics including dissociation and recombination reactions.

When considering molecules with internal degrees of freedom (DOF), it is necessary to consider the possibility of inelastic collisions and allow the energies of the colliding molecules to be redistributed among the available translational and internal modes. The redistribution is usually based on an approach toward an equilibrium distribution among the available modes. This approach originates from the Borgnakke-Larsen (BL) method published in 1975, and allows molecular models (hard sphere, variable hard sphere, variable soft sphere, etc.) to mathematically mimic the physically significant aspects of real molecules. ${ }^{28}$ Our phenomenological model is based on the continuous model of Bird, ${ }^{18}$ an approach that obeys detailed balancing. The method selects the post-collision internal energies by sampling from the known equilibrium distributions associated with a "temperature" based on the energy specified in the collision. It is important to note that the energy exchanges are completed for each accepted molecule independent of its colliding partner. In general, the redistribution of energy considers first any available vibrational modes, and then rotation, then the remainder is redistributed as translation energy. The relaxation of energy is a convenient example of this phenomenological method and it is shown in Fig. 8 for a diatomic chlorine molecule $\left(\mathrm{Cl}_{2}\right)$ with a fixed relaxation collision number $(\Lambda)$ and two rotational degrees of freedom. The conditions for the simulation corresponding to the results shown in Fig. 8 are listed in Table 1. In this simple example it may be seen that the energy exchange method implemented adheres exceptionally well to predicted rates of a basic model assuming classical internal degrees of freedom and constant relaxation collision numbers. The model rates shown in Fig. 8 are based on expressions given by Bird ${ }^{17}$ for rotational temperature:

$$
T_{\text {rot }}=F_{s} T_{\text {equil }}+F_{u} T_{r o t, 0}
$$

where $T_{\text {rot }}$ is the final rotational temperature, $T_{\text {rot }, 0}$ is the initial rotational temperature, $T_{\text {equil }}$ is the equilibrium temperature, and $\mathrm{F}_{\mathrm{s}}$ and $\mathrm{F}_{\mathrm{u}}$ are the fractions of selected and unselected molecules for energy exchange, respectively. The theoretical expression is given as

$$
T_{\text {rot }}=T_{\text {equil }}-\left(T_{\text {equil }}-T_{\text {rot }, 0}\right)(1-1 / N)^{N \nu \Lambda t},
$$

where $v$ is the collision rate per molecule, $\Lambda$ is the fraction of inelastic collisions for rotation, and $N v \Lambda t$ is the number of selections from $N$ total molecules after a time $t$.

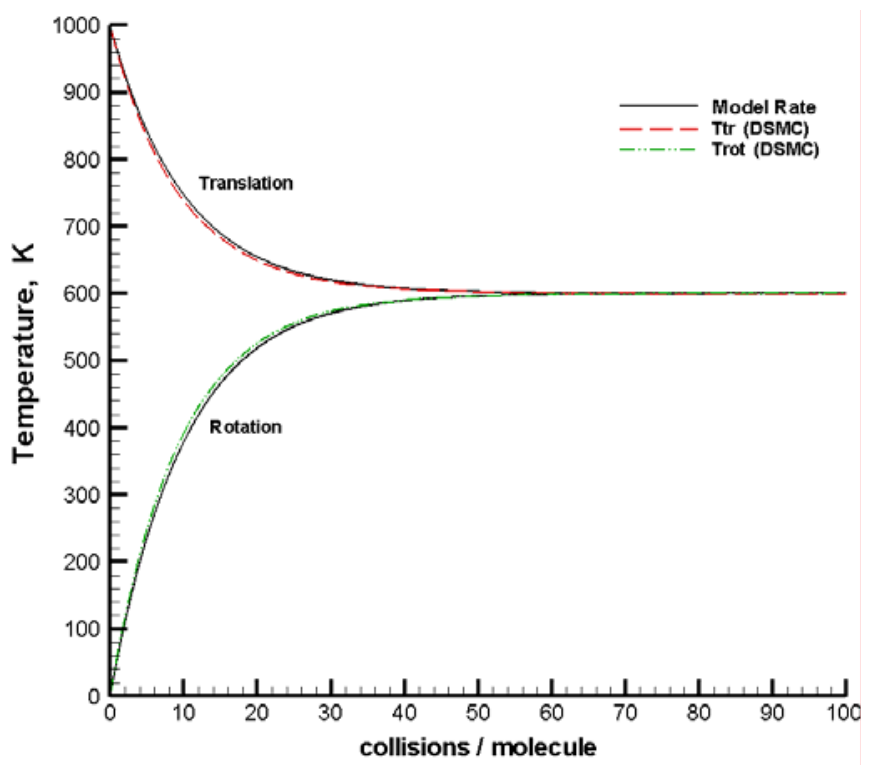

Table 1. Classical Relaxation Properties

\begin{tabular}{lc} 
Molecule Properties & \\
Species & $\mathrm{Cl}_{2}$ \\
Rotational DOF & 2 \\
Number of molecules & 30,000 \\
Collision number & 10 \\
& \\
Cell Properties & \\
Number of cells & 1 \\
Initial Temperature & $1000 \mathrm{~K}$ \\
Cell size / $\lambda$ & 0.25 \\
$d t /$ mean collision time & 0.025 \\
Number of time steps & 5000 \\
Number of ensembles & 10 \\
\hline
\end{tabular}

Figure 8. DSMC simulation of hard sphere molecules undergoing classical rotational relaxation. 
A discrete energy model was also developed to model systems with quantized energy levels, as for electronic states and widely spaced vibrational states. This model is based primarily on the methods developed by Anderson et al. ${ }^{29}$ and Boyd $^{30}$ in order to more accurately represent the internal structure of diatomic and polyatomic molecules. This quantum exchange procedure is more accurate than the classical $\mathrm{BL}$ method and takes into account characteristic vibrational temperatures $\left(\Theta_{\mathrm{v}}\right)$ and collision energies to determine the populations of energy levels for a given species. A similar test to that of Fig. 8 was completed to determine to what extent the discrete exchange method has on different molecular species. Hydrogen and chlorine molecules were simulated allowing only a single excited vibrational level. The significant difference between these two molecules is the disparity in the characteristic vibrational temperatures, where $\Theta_{v}$ for $\mathrm{H}_{2}$ is more than 7.5 time greater than that for $\mathrm{Cl}_{2}$ (as shown in Table 2). The results shown in Figs. 9 and 10 were simulated under the same conditions, and varied only by the properties of the molecules being simulated. Figures 9 and 10 were compared to the same theoretical expression for relaxation given in Eqn. (2). Therefore, when assuming $N>>1$ and $N v \Lambda t \gg$ 1, Eqn. (2) can be approximated as

$$
T_{\text {rot }}=T_{\text {equil }}-\left(T_{\text {equil }}-T_{\text {rot }, 0}\right) \exp (-v \Lambda t) .
$$

Assuming no quantum events, Eqn. (3) defines the classical relaxation (as in Fig. 8). Figures 9 and 10 show the deviation from Eqn. (3) when using a discrete vibrational energy exchange. There is a large amount of scatter in the vibrational temperature in Fig. 9 because the temperature is based on the population of molecules at the first level relative to that at the zeroth level. Since energy of the first level is low (based on a characteristic temperature of 801 $\mathrm{K})$ relative to the energy available to the system, higher levels will have significant numbers of molecules. This is the same justification for the rapid increase in vibrational temperature for hydrogen, given its characteristic temperature of $6159 \mathrm{~K}$ and the initial freestream temperature of $4000 \mathrm{~K}$, a majority of the molecules are located at the first level, reducing the scatter and causing the rapid increase in vibrational temperature.

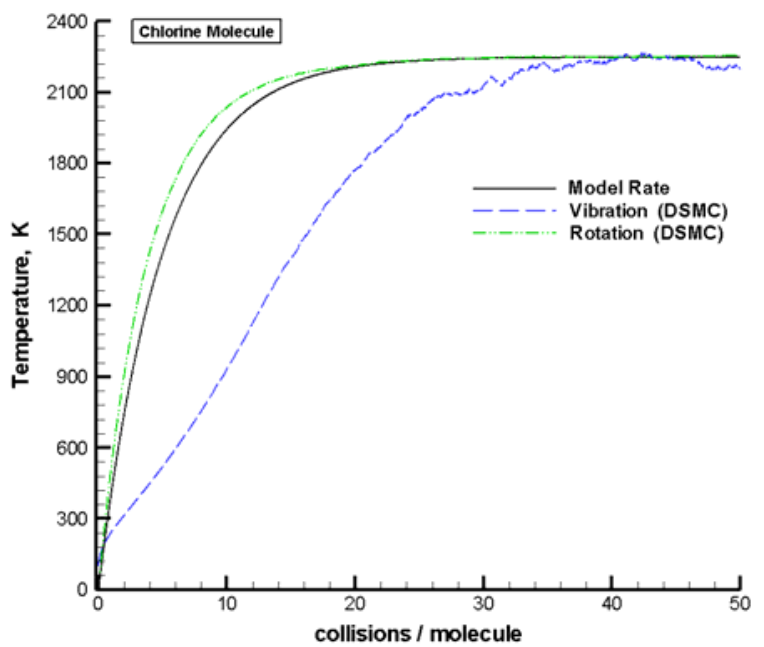

Figure 9. DSMC simulation of $\mathrm{Cl}_{2}$ molecules undergoing discrete vibrational relaxation using a quantum Borgnakke-Larsen method.

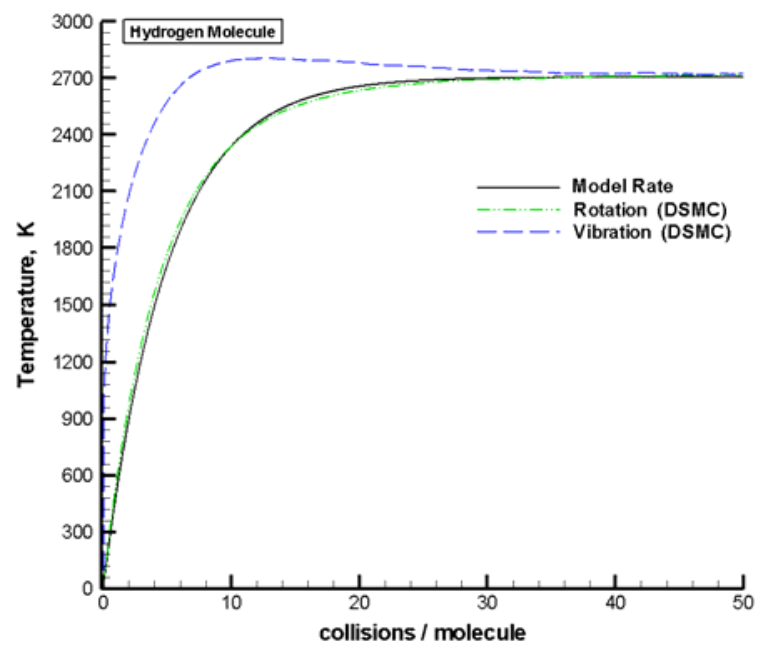

Figure 10. DSMC simulation of $\mathrm{H}_{2}$ molecules undergoing discrete vibrational relaxation using a discrete Borgnakke-Larsen method. 
The reaction model developed is capable of simulating reactions based on experimental rate data for particles with and without internal degrees of freedom. Full cross-section and line-of-centers reaction models are available to simulate hard sphere reaction dynamics for molecules with a specified number of square terms. The algorithm is capable of simulating any number of molecules with real molecular properties (molecular mass, diameter, degrees of freedom, etc.) for any number of reactions based on the experimental data fitted to the Arrhenius equation. In most earlier work, rate expressions involving series of terms $E_{a} / k T$ in increasing powers were approximated. We found it necessary to include all terms for accurate representations. For example, the rate expression for the full cross-section model for an even integer number of square terms can be summarized as

$$
k(T)=S(8 k T / \pi \mu)^{1 / 2} \exp \left(-E_{a} / k T\right)\left[\sum_{n=0,2,4, . .}^{s+2} \frac{\left(E_{a} / k T\right)^{\frac{n}{2}}}{\Gamma\left(\frac{n}{2}+1\right)}\right]
$$

where $\Gamma(\mathrm{m})=(\mathrm{m}-1) !, S$ is the reaction cross section, $(8 k T / \pi \mu)^{1 / 2}$ is the mean velocity, $E_{a}$ is the activation energy for reaction, $k$ is the Boltzmann constant, $T$ is the temperature, and $s$ is the total number of square terms for the reactive pair. ${ }^{31}$

Dissociations are treated as reactions in the same way as other bimolecular encounters and simulate experimental rates measured for the excitation of a molecule exceeding its characteristic dissociation energy. When dissociation occurs, a new molecule is added to the flow field within the cell containing the dissociation. All remaining energy after the endothermic reaction is redistributed among the separating particles and the particles are assigned random post-collision velocities. The total number of molecules is updated to account for the new atom, and the process is again repeated after any future dissociations.

At this stage of development we found it convenient to treat the termolecular recombination reactions with analytical methods with all other reactions including, most importantly, the chain initiation and propagation reactions treated stochastically. The treatment of recombinations is based on equilibrium collision theory. The number of recombinations is calculated per unit volume per unit time as a function of the macroscopic cell temperature and individual species concentrations. A separate number of recombinations are calculated for each colliding pair, the particles are chosen at random, and the associated recombination reaction for that pair is decremented by unity. The relative energy of the pair is calculated and they are combined to form a single molecule. The velocity of the new molecule is set to the center-of-mass velocity of the original pair. The required "third body" is then chosen under the conditions of the reaction and undergoes a collision with the newly formed molecule to simulate stabilization. A redistribution of energy is allowed using the Borgnakke-Larsen method.

\section{Results}

This section discusses the simulations conducted with the prototypical hydrogen-chlorine reaction system. Many of the DSMC algorithm modifications discussed in the prior section were implemented to model the five-step kinetic mechanism given by H. Guénoche et al. ${ }^{32}$ The gaseous system reacts by the Nernst chain reaction mechanism based on five species and 34 possible reactions, 30 of which are dissociations and recombinations. Table 3 lists the mechanism given by $\mathrm{H}$. Guénoche et al., and was modified slightly for the DSMC simulations. The reaction rates not specified in Table 3 were calculated using those given and their corresponding equilibrium constants.

Table 3. Hydrogen-Chlorine reduced kinetic mechanism.

\begin{tabular}{|c|c|c|c|c|c|c|}
\hline & Kinetic Model & $\mathrm{k}=A T^{\eta} \exp \left(-E^{*} / \mathrm{RT}\right)$ & A & $\mathrm{n}$ & $\mathrm{E}^{*}$ & M \\
\hline 1 & $\mathrm{H}+\mathrm{Cl}_{2} \leftrightarrow \mathrm{HCl}+\mathrm{Cl}$ & $\mathrm{k}_{+1}$ & $6.61 \times 10^{11}$ & 0.68 & 1090 & \\
\hline 2 & $\mathrm{Cl}+\mathrm{H}_{2} \leftrightarrow \mathrm{HCl}+\mathrm{H}$ & $\mathrm{k}_{+2}$ & $4.8 \times 10^{13}$ & 0 & 5260 & \\
\hline 3 & $\mathrm{HCl}+\mathrm{M} \leftrightarrow \mathrm{H}+\mathrm{Cl}+\mathrm{M}$ & $\mathrm{k}_{+3, \mathrm{M}}$ & $6.76 \times 10^{21}$ & -2.0 & 102,170 & $\mathrm{H}_{2}, \mathrm{Cl}_{2}, \mathrm{HCl}, \mathrm{H}, \mathrm{Cl}$ \\
\hline 4 & $\mathrm{Cl}_{2}+\mathrm{M} \leftrightarrow \mathrm{Cl}+\mathrm{Cl}+\mathrm{M}$ & $\mathrm{k}_{+4, \mathrm{M}}$ & $\begin{array}{l}6.15 \times 10^{21} \\
6.15 \times 10^{22}\end{array}$ & $\begin{array}{l}-2.07 \\
-2.07\end{array}$ & $\begin{array}{l}57,040 \\
57,050\end{array}$ & $\begin{array}{l}\mathrm{H}_{2}, \mathrm{Cl}_{2}, \mathrm{HCl}, \mathrm{H} \\
\mathrm{Cl}\end{array}$ \\
\hline 5 & $\mathrm{H}_{2}+\mathrm{M} \leftrightarrow \mathrm{H}+\mathrm{H}+\mathrm{M}$ & $\mathrm{k}_{-5, \mathrm{M}}$ & $\begin{array}{l}1.0 \times 10^{18} \\
2.0 \times 10^{19} \\
9.0 \times 10^{16}\end{array}$ & $\begin{array}{l}-1.0 \\
-1.0 \\
-0.6\end{array}$ & $\begin{array}{l}0 \\
0 \\
0\end{array}$ & $\begin{array}{l}\mathrm{HCl}, \mathrm{Cl}_{2}, \mathrm{Cl} \\
\mathrm{H} \\
\mathrm{H}_{2}\end{array}$ \\
\hline
\end{tabular}


DSMC simulations were completed for both free-radical and thermal ignitions of the hydrogen-chlorine system. The thermal ignition simulations began with $10,000 \mathrm{H}_{2}$ and $10,000 \mathrm{Cl}_{2}$ molecules initialized at a temperature of $2500 \mathrm{~K}$. This caused the immediate dissociation of $\mathrm{Cl}_{2}$ molecules and led to the rapid production of $\mathrm{HCl}$ due to the chain-propagating $\mathrm{H}+\mathrm{Cl}_{2}$ and $\mathrm{Cl}+\mathrm{H}_{2}$ reactions. These reactions occurred so rapidly that the exchange of vibrational energy could not maintain equilibrium energy as shown in Fig. 11. Figure 12 displays the number of particles of each species throughout the explosion. The mechanism took less than 6 microseconds to fully exhaust all $\mathrm{H}_{2}$ and $\mathrm{Cl}_{2}$ molecules and after which it relied solely on the dissociation of $\mathrm{HCl}$ and recombinations of $\mathrm{H}$ and $\mathrm{Cl}$ atoms to maintain the system. The simulation of the thermal ignition was completed over five ensembles, each requiring approximately 35 minutes on a $3.6 \mathrm{GHz}$ Pentium 4 processor to simulate the reaction of 20,000 molecules over 10 microseconds. The time step size was approximately $10^{-10}$ seconds with 100,000 time steps recorded.

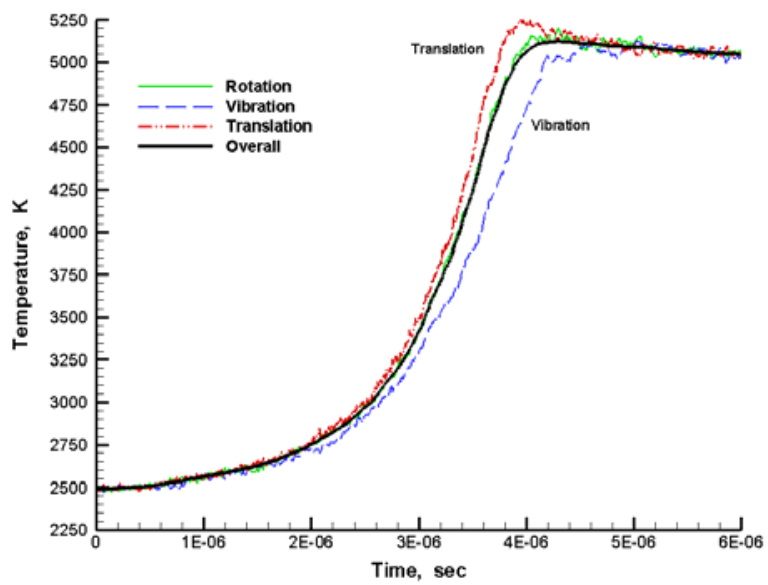

Figure 11. Nonequilibrium temperature profile for hydrogen-chlorine explosion after thermal ignition at $2500 \mathrm{~K}$.

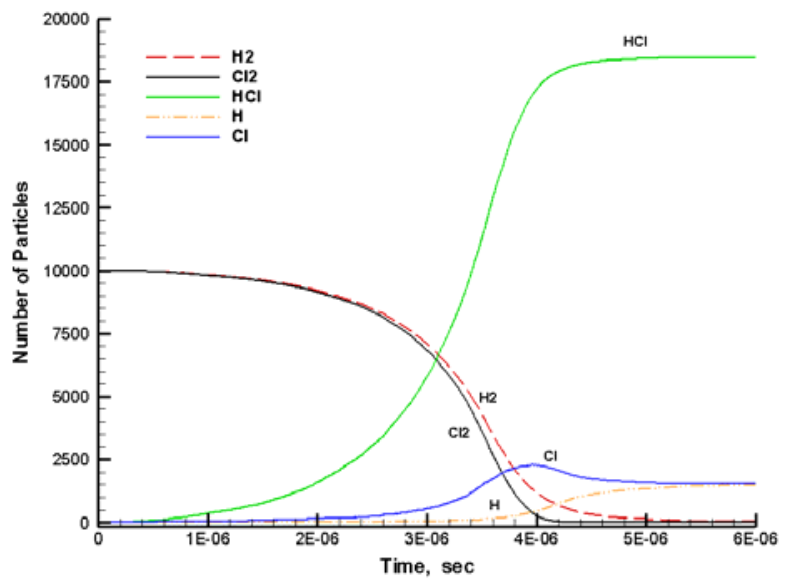

Figure 12. Species profiles after thermal ignition of the hydrogen-chlorine system at $2500 \mathrm{~K}$.

The free-radical ignition simulations were run at an initial temperature of $1000 \mathrm{~K}$ with a total of 2001 molecules. A single hydrogen atom was introduced randomly into a system of $1000 \mathrm{H}_{2}$ and $1000 \mathrm{Cl}_{2}$ molecules initiating and sustaining a chain reaction until there was enough energy in the system to produce dissociations. A temperature of $1000 \mathrm{~K}$ was chosen because it is low enough to avoid spontaneous dissociation reactions, but high enough to prevent the slow, time-consuming, and often unpredictable series of reactions required if the system were initialized at 298.15 K. Simulations run at $298.15 \mathrm{~K}$ presented no guarantee that a free-radical would initiate an explosion. Some simulations would not produce a single reaction, while others over the same length of time would generate tens or hundreds of reactions. The outcome depended entirely on the random initialization of the particles and on the random selection of collision partners. Figure 13 displays the number of dissociations and recombinations, and temperature for the DSMC simulation at $1000 \mathrm{~K}$. The free radical effect is displayed in delay of the initiation of dissociation and recombination reactions relative to the temperature profile. The simulation required roughly 12 microseconds and over 500 freeradical reactions before the first dissociation was recorded. Recombinations were not found until essentially all $\mathrm{H}_{2}$ and $\mathrm{Cl}_{2}$ molecules were reacted. The DSMC simulation used 200,000

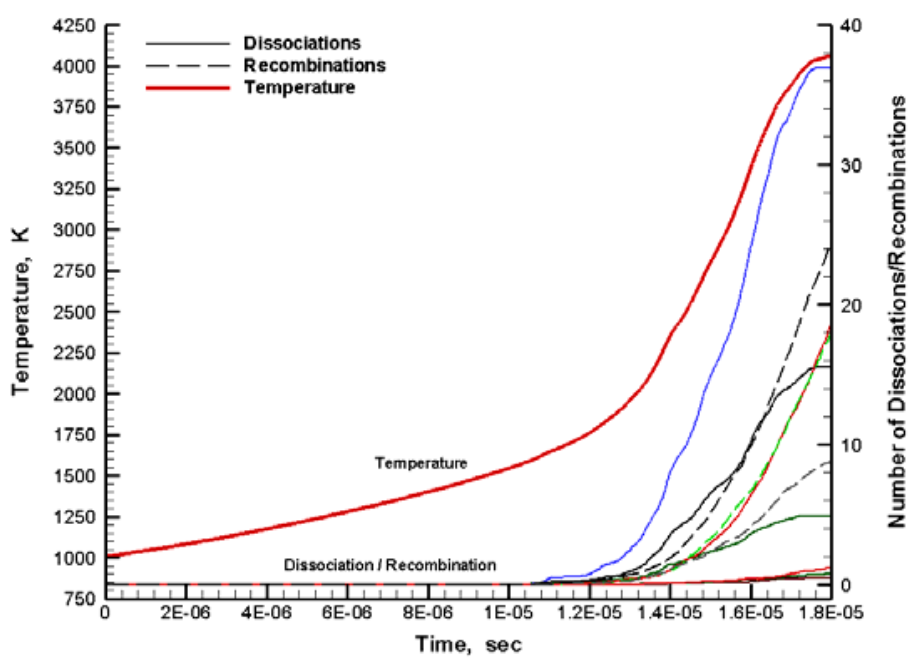

Figure 13. Dissociation and recombination rates relative to overall temperature after free-radical ignition at $1000 \mathrm{~K}$. 
time steps to simulate 19 microseconds. Ninety ensembles were completed over 15 hours on a $3.6 \mathrm{GHz}$ Pentium 4 processor for a total of 180,090 molecules simulated.

\section{Conclusion}

We have found the DSMC method is capable of directly simulating detonations in a level of detail not possible with continuum (e.g., Euler or Navier-Stokes) approaches. The recent modifications to the DSMC algorithm described in this paper have allowed us to simulate complex chemical kinetics with the goal of modeling "real" detonations. With future computers capable of petaflop performance, it will be possible to perform 3-D simulations of the detonation process in order to study multidimensional detonation front instability, ignition, as well as temperature fluctuations due to both irregularities in shock speed (over the entire shock front) and transverse (counter propagating) shock waves.

\section{Acknowledgments}

We thank the National Science Foundation for funding the IGERT Consortium for Education in Many-Body Applications (CEMBA) and its continued support of this research (NSF DGE-9987589). We also gratefully acknowledge support from the Office of Naval Research (ONR N00014-05-1-0844).

\section{References}

${ }^{1}$ L. N. Long and J. B. Anderson, "The simulation of detonations using a Monte Carlo method," Rarefied Gas Dynamics, $22^{\text {nd }}$ International Symposium, edited by T. J. Bartel and M. A. Gallis, AIP, New York, Vol. 585, CP585, pp. 653-657, 2001.

${ }^{2}$ J. B. Anderson and L. N. Long, "Direct Simulation of Pathological Detonations," Rarefied Gas Dynamics, $23^{\text {rd }}$ International Symposium, edited by A. Ketsdever and P. Muntz, AIP, New York, Vol. 663, CP663, pp. 186-193, 2003.

${ }^{3}$ J. B. Anderson and L. N. Long, "Direct Monte Carlo simulation of chemical reaction systems: Prediction of ultrafast detonations," Journal of Chemical Physics, Vol. 118, No. 7, pp. 3102-3110, 2003.

${ }^{4}$ P. D. O'Connor, J. B. Anderson and L. N. Long, "Direct Simulation of Ultrafast Detonations in Mixtures," Rarefied Gas Dynamics, $24^{\text {th }}$ International Symposium, edited by M. Capitelli, AIP, New York, Vol. 762, pp. 517-522, 2005.

${ }^{5} \mathrm{E}$. Mallard and H. L. Le Chatelier, "On the propagation velocity of burning in gaseous explosive mixtures," Comp. Rend. Hebd. Séances Acad. Sci., Vol. 93, pp. 145-148, 1881.

${ }^{6} \mathrm{M}$. Berthelot and P. Vieille, "On the velocity of propagation of explosive processes in gases," Comp. Rend. Hebd. Séances Acad. Sci., Vol. 93, pp. 18-21, 1881.

${ }^{7}$ D. L. Chapman, "On the rate of explosion in gases," Philos. Mag., Vol. 47, pp. 90-104, 1899.

${ }^{8}$ E. Jouguet, "On the propagation of chemical reactions in gases," J. de Mathématique Pures et Appliquées, Vol. 1, 347-425, 1905; Vol. 2, pp. 5-85, 1906.

${ }^{9}$ Ya. B. Zel'dovich, "On the theory of the propagation of detonation in gaseous systems," Zh. Eksp. Teor. Fiz., Vol. 10, pp. 542-568, 1940.

${ }^{10}$ J. von Neumann, "Theory of detonation waves," Off. Sci. Res. Dev. Rep. 549, Balist. Res. Lab. File No. X-122, Aberdeen Proving Ground, MD, 1942.

${ }^{11}$ W. Döring, "Über den Detonationsvorgang in Gasen," Annalen der Physik, Vol. 435, No. 6-7, pp. 421-436, 1943.

${ }^{12}$ J. O. Hirschfelder and C. F. Curtiss, "Theory of Detonations. I. Irreversible Unimolecular Reaction," The Journal of Chemical Physics, Vol. 28, No. 6, pp. 1130-1147, 1958.

${ }^{13}$ S. Chapman and T. G. Cowling, The Mathematical Theory of Non-Uniform Gases, $3^{\text {rd }}$ ed., Cambridge University Press, Great Britain, 1991.

${ }^{14}$ L.N. Long, "Navier-Stokes and Monte Carlo Results for Hypersonic Flows," AIAA Journal, Vol. 29, No. 2 , Feb., 1991.

${ }^{15}$ L.N. Long, M. Kamon, T, Chyczewski, J. Myczkowski, "A Deterministic Parallel Algorithm to Solve a Model Boltzmann Equation (BGK),” Computing Systems in Engineering Journal, Vol. 3, No. 1-4, Dec., 1992.

${ }^{16}$ G. A. Bird, "Direct simulation and the Boltzmann equation," Physics of Fluids, Vol. 13, pp. 2676-2687, 1970.

${ }^{17}$ G. A. Bird, “Approach to Translational Equilibrium in a Rigid Sphere Gas,” Physics of Fluids, Vol. 6, pp. 1518-1519, 1963.

${ }^{18}$ G. A. Bird, Molecular Gas Dynamics and the Direct Simulation of Gas Flows, $2^{\text {nd }}$ ed., Oxford Science Publications, Clarendon, Oxford, 1994.

${ }^{19}$ K. C. Kannenberg and I. D. Boyd, "Three Dimensional Monte Carlo Simulations of Plume Impingement," Journal of Thermophysics and Heat Transfer, Vol. 13, pp. 226-235, 1999.

${ }^{20}$ K. Boyles, G. LeBeau, F. Lumpkin, and R. Blanchard, "The Use of Virtual Sub-cells in DSMC Analysis of Orbiter Aerodynamics at High Altitudes Upon Reentry," 41 $1^{\text {st }}$ Aerospace Sciences Meeting and Exhibit, CP 1030, 2003.

${ }^{21}$ A. Sharma and L. N. Long, "Numerical simulation of the blast impact problem using the Direct Simulation Monte Carlo (DSMC) method," Journal of Computational Physics, Vol. 200, pp. 211-237, 2004.

${ }^{22}$ J. V. Austin and D. B. Goldstein, "Direct Numerical Simulation of Circumplanetary Winds on Io," Bulletin of the American Astronomical Society, Vol. 29, No. 3, pp. 1004, 1997. 
${ }^{23}$ F. Alexander, A. Garcia, and B. Adler, "Direct Simulation Monte Carlo for Thin Film Bearings," Phys. Fluids, Vol. 6, 3854, 1994.

${ }^{24}$ Q. Sun and I. D. Boyd, “A Direct Simulation Method for Subsonic, Micro-Scale Gas Flows,” Journal of Computational Physics, Vol. 119, No. 2, pp. 400-425, July, 2002.

${ }^{25}$ A. Danforth and L. N. Long, "Nonlinear Acoustic Simulations using Direct Simulation Monte Carlo," Journal of the Acoustical Society of America, Vol. 116, No. 4, pp. 1948-1955, 2004.

${ }^{26}$ A. Danforth-Hanford, P. O’Connor, L. Long, and J. Anderson, "Molecular Relaxation Simulations in Nonlinear Acoustics using Direct Simulation Monte Carlo," Innovations in Nonlinear Acoustics, edited by A. Atchley, V. Sparrow and R. Keolian, Vol. 838, AIP, New York, pp. 556-559, 2005.

${ }^{27}$ J. G. Kirkwood and W. W. Wood, "Structure of a Steady-State Plane Detonation Wave with Finite Reaction Rate," Journal of Chemical Physics, Vol. 22, pp. 1915-1919, 1954.

${ }^{28}$ C. Borgnakke and P. Larsen, "Statistical Collision Model for Monte Carlo Simulation of Polyatomic Gas Mixture," Journal of Computational Physics, Vol. 18, pp. 405-420, Jan., 1975.

${ }^{29}$ J. B. Anderson, J. D. Foch, M. J. Shaw, R. C. Stern and B. J. Wu, "Statistical theory of electronic energy relaxation," Rarefied Gas Dynamics, $15^{\text {th }}$ International Symposium, edited by V. Boffi and C. Cercignani, B. G. Teubner Stuttgart, Vol. II, pp. 413-421, 1986.

${ }^{30}$ F. Bergemann and I. D. Boyd, "New Discrete Vibrational Energy Model for the Direct Simulation Monte Carlo Method,"

Rarefied Gas Dynamics, $18^{\text {th }}$ International Symposium, edited by B. Shizgal, Progress in Astronautics and Aeronautics, AIAA, California, Vol. 160, pp. 174-183, 1994.

${ }^{31}$ P. D. O'Connor, L. N. Long, and J. B. Anderson, “Exact theoretical rates for modern kinetic systems," Submission pending to Chemical Physics Letters, July, 2006.

${ }^{32}$ H. Guénoche, P. Le Diuzet, and C. Sèdes, "Influence of the Heat-Release Function on the Detonation States," Progress in Astronautics and Aeronautics, AIAA, New York, Vol. 75, pp. 387-407, 1981. 